\title{
Dynamic Range Adaptation to Spectral Stimulus Statistics in Human Auditory Cortex
}

\author{
Björn Herrmann, Nadine Schlichting, and Jonas Obleser \\ Max Planck Research Group “Auditory Cognition” at the Max Planck Institute for Human Cognitive and Brain Sciences, 04103 Leipzig, Germany
}

Classically, neural adaptation refers to a reduction in response magnitude by sustained stimulation. In human electroencephalography (EEG), neural adaptation has been measured, for example, as frequency-specific response decrease by previous stimulation. Only recently and mainly based on animal studies, it has been suggested that statistical properties in the stimulation lead to adjustments of neural sensitivity and affect neural response adaptation. However, it is thus far unresolved which statistical parameters in the acoustic stimulation spectrum affect frequency-specific neural adaptation, and on which time scales the effects take place. The present human EEG study investigated the potential influence of the overall spectral range as well as the spectral spacing of the acoustic stimulation spectrum on frequency-specific neural adaptation. Tones randomly varying in frequency were presented passively and computational modeling of frequency-specific neural adaptation was used. Frequency-specific adaptation was observed for all presentation conditions. Critically, however, the spread of adaptation (i.e., degree of coadaptation) in tonotopically organized regions of auditory cortex changed with the spectral range of the acoustic stimulation. In contrast, spectral spacing did not affect the spread of frequency-specific adaptation. Therefore, changes in neural sensitivity in auditory cortex are directly coupled to the overall spectral range of the acoustic stimulation, which suggests that neural adjustments to spectral stimulus statistics occur over a time scale of multiple seconds.

Key words: dynamic range adaptation; frequency-specific adaptation; stimulus statistics

\section{Introduction}

A key feature of natural environments is the dynamic change with which they confront the neural system. It has been proposed that efficient coding of environmental changes requires the neural system to adapt to the properties of the stimulation distribution (Fairhall et al., 2001; Wark et al., 2007). However, adaptation of neural activity can be separated into two types: stimulus-specific adaptation and adaptation to stimulus statistics. Stimulusspecific adaptation relates to reduced neural responsiveness due to sustained stimulation (Ulanovsky et al., 2003, 2004; Bäuerle et al., 2011; Taaseh et al., 2011). In the context of auditory spectral stimulation, it is also referred to as frequency-specific adaptation (Herrmann et al., 2013a, 2013b). Adaptation to stimulus statistics, on the other hand, relates to adjustments in neuronal firing to the mean and variance of a stimulus distribution such that the dynamic range of the neuron's response sensitivity matches the statistical properties of the stimulation (Brenner et al., 2000; Kvale and Schreiner, 2004; Dean et al., 2008; Watkins and Barbour, 2008; Dahmen et al., 2010, Rabinowitz et al., 2011). Critically, although frequency-specific adaptation has

\footnotetext{
Received Sept. 17, 2013; revised Nov. 15, 2013; accepted Nov. 22, 2013.

Author contributions: B.H., N.S., and J.O. designed research; B.H. and N.S. performed research; B.H. and N.S. analyzed data; B.H. and J.O. wrote the paper.

This work was supported by the Max Planck Society. B.H. and J.0. are supported by a Max Planck Research Group grant to J.0. We thank Elizabeth Kelly for proofreading the manuscript and two anonymous reviewers for helpful comments.

This article is freely available online through the J Neurosci Author Open Choice option.

Correspondence should be addressed to Björn Herrmann, Max Planck Institute for Human Cognitive and Brain Sciences, Stephanstraße 1a, Leipzig 04103, Germany. E-mail: bherrmann@cbs.mpg.de.

DOI:10.1523/JNEUROSCI.3974-13.2014

Copyright $\odot 2014$ the authors $\quad 0270-6474 / 14 / 340327-05 \$ 15.00 / 0$
}

been investigated in animals and humans, dynamic range adaptation in audition has mainly been investigated using animal electrophysiology and adjustments to sound-level distributions (Dean et al., 2005; Wen et al., 2009, 2012).

Observations of frequency-specific adaptation in humans are based on electroencephalography (EEG) and event-related potentials. Modulations of the auditory N1 component, a negative deflection maximal at $80-120 \mathrm{~ms}$ after stimulus onset and localized to auditory cortices (Maess et al., 2007), show strong similarities to frequency-specific neural adaptation reported for animals (Butler, 1968; Nätänen et al., 1988; Ulanovsky et al., 2003; Taaseh et al., 2011; Herrmann et al., 2013a). Specifically, the N1 magnitude is sensitive to stimulation history, including interstimulus duration and spectral distance to preceding acoustic stimuli (Näätänen et al., 1988; Sams et al., 1993; McEvoy et al., 1997; Herrmann et al., 2013a).

Importantly, spectral properties in the acoustic stimulation influence the spread of adaptation (i.e., degree of coadaptation) in tonotopically organized regions of auditory cortex and thereby affect frequency-specific N1 adaptation (Herrmann et al., 2013a, $2013 \mathrm{~b}$ ). In these previous studies, however, it remained unresolved whether dynamic range changes in neural response sensitivity are linked to the overall spectral range in the stimulation or to the spectral spacing within the stimulation. Therefore, the present EEG study aimed to examine which of these two statistical parameters in the acoustic stimulation spectrum drives dynamic range adaptation in human auditory cortex. Furthermore, resolving this question provides information about the time scale of dynamic range adaptation - that is, whether adaptation to stimulus statistics occurs on a time scale of multiple seconds (captured by overall spectral range) or hundreds of milliseconds 


\section{A Experimental stimulation}

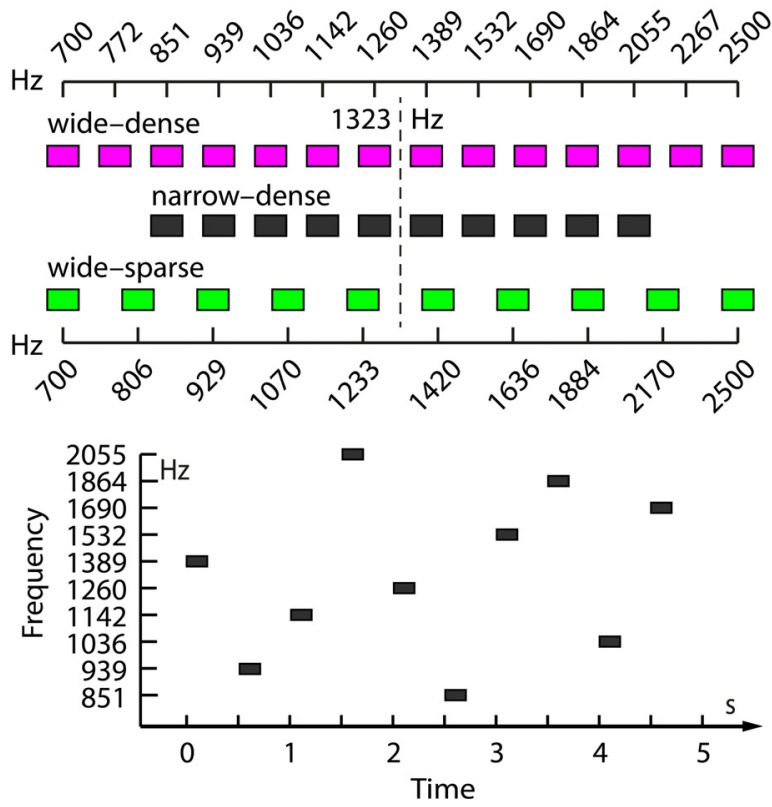

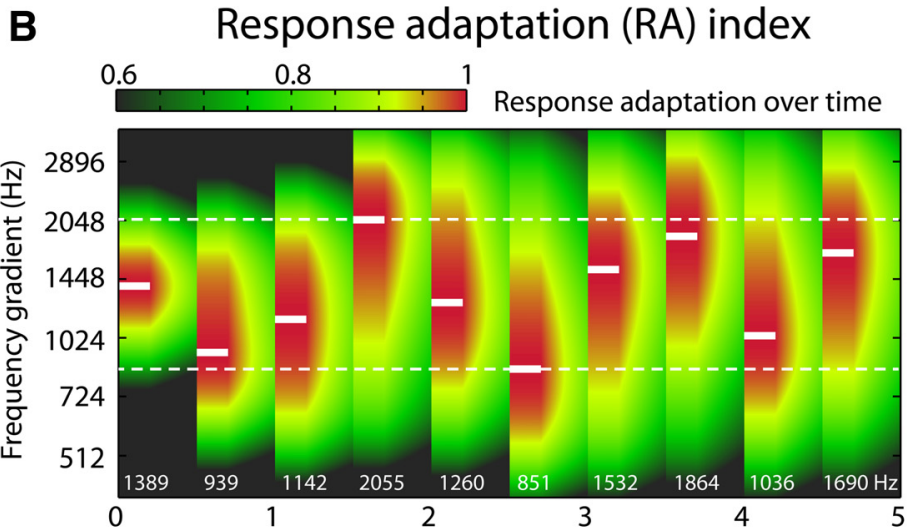
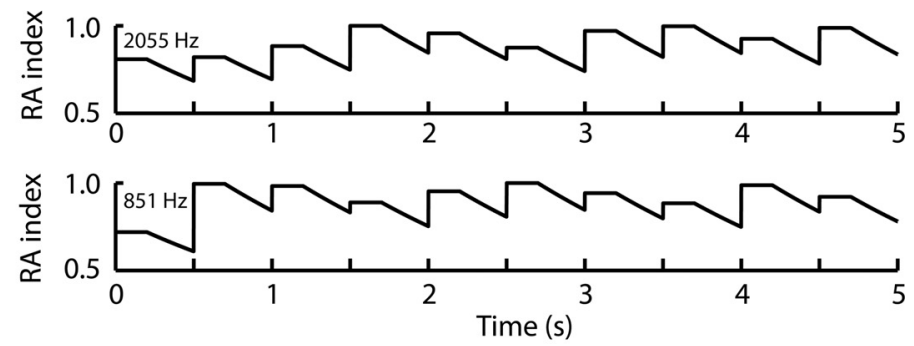

Figure 1. Experimental stimulation and expected response adaptation. $\boldsymbol{A}$, Top: Tone frequencies for each condition. Bottom: Example of random tone presentation for the narrow-dense condition. $\boldsymbol{B}$, Example of expected response adaptation over time for the narrow-dense condition ( $\tau=1.8 \mathrm{~s}, \sigma=0.87 \mathrm{SD})$. Top: A $200 \mathrm{~ms}$ tone is presented every $0.5 \mathrm{~s}$ ( $x$-axis). White lines and numbers inside the graph indicate the presented tone frequency. The $y$-axis displays the frequency gradient of auditory cortex along which adaptation spread ( $\sigma$ ) occurs. RA indices (color-coded; red $=$ full adaptation) increase at tone onset and decrease after tone offset (recovery). Bottom: Time course of RA indices for two frequencies (white dashed lines, top).

(captured by trial-by-trial spectral spacing). We focused on $\mathrm{N} 1$ responses and used a computational model that incorporates recovery from adaptation over time and spread of adaptation along tonotopically organized regions of auditory cortex to predict neural response magnitude.

\section{Materials and Methods}

Participants. Twenty-six healthy adults (mean age: 25.2 years; 13 female) participated in the present study. Participants were right-handed and had no self-reported hearing problems or history of neurological diseases. They gave written informed consent and were paid 7 Euro per hour. The study was conducted in accordance with the Declaration of Helsinki and was approved by the local ethics committee of the University of Leipzig.

Acoustic stimulation. Stimuli consisted of logarithmically spaced sinusoidal tones (200 ms duration, $10 \mathrm{~ms}$ rise/fall times) in three conditions (Fig. 1A). In detail, tone frequencies were either spaced densely with a wide range (wide-dense; range: $700-2500 \mathrm{~Hz}, \log 2$-spacing: 0.1413), were spaced densely with a narrow range (narrow-dense; range: $851-$ $2055 \mathrm{~Hz}, \log 2$-spacing: 0.1413), or were spaced sparsely with a wide range (wide-sparse; range: $700-2500 \mathrm{~Hz}, \log 2$-spacing: 0.2041).

Procedure. Electroencephalograms were recorded in an electrically shielded and sound-attenuated booth while participants were presented with the acoustic stimulation via headphones (HD 25-SP II; Sennheiser). At the beginning of the experiment, the participant's individual hearing threshold was determined for the centrally located $1323 \mathrm{~Hz}$ tone using the method of limits (Leek, 2001). Acoustic stimulation was then presented at $60 \mathrm{~dB}$ above the individual hearing threshold.

Experimental stimulation was presented in nine blocks (two of which were a control condition not reported here) while participants watched a silent self-chosen movie with subtitles. Two blocks of presentation were performed for each of the three conditions. The order of presentation was randomized for each participant. In each block, acoustic stimulation started after a silent period of $>10 \mathrm{~s}$ to ensure full responsiveness of the neural populations (Sams et al., 1993). Within each block, the unique tone frequencies of one condition were presented in 115 trains (i.e., 115 passes through all unique tone frequencies of the condition) without pauses between trains. Tone frequencies within each train were random- ized and no two consecutive tones ever had the same frequency, even during transitions between trains (Fig. 1A). Tones were presented with an onset-to-onset interval of $0.5 \mathrm{~s}$. In the last block presented to each participant, the $1323 \mathrm{~Hz}$ tone was presented 30 times with an onset-toonset interval of $10 \mathrm{~s}$ (no-adaptation condition).

Data acquisition and preprocessing. Signals were recorded from $26 \mathrm{Ag} /$ Ag-Cl scalp electrodes (Easycap) and additionally from left and right mastoids, nose (online reference), and ground (at the sternum). The sampling rate was $500 \mathrm{~Hz}$ (TMS international; $135 \mathrm{~Hz}$ low-pass filter; impedances $<5 \mathrm{k} \Omega$ ).

Data were analyzed using Fieldtrip software (v20110527; Oostenveld et al., 2011) and custom MATLAB scripts (MathWorks). Recordings were rereferenced to the linked mastoids, high-pass filtered at $0.5 \mathrm{~Hz}$ (1747 points, Hamming window; $>120$ dB DC suppression to replace baseline correction, Ruhnau et al., 2012), low-pass filtered at $80 \mathrm{~Hz}$, and down-sampled to $250 \mathrm{~Hz}$. Signals time locked to tone onsets (-1.6-1.9 s) were corrected for artifacts (eye movements, heart activity, noisy channels) using independent components analysis. Subsequently, epochs containing a signal range larger than $120 \mu \mathrm{V}$ in any EEG electrode were excluded. Epochs were low-pass filtered at $20.1 \mathrm{~Hz}$ and redefined for data analysis ranging from -0.1 to $0.4 \mathrm{~s}$. One participant was excluded at this stage because no N1 response in any condition was discernible.

Data processing: model of frequency-specific adaptation. The present study aimed to investigate how stimulus statistics in the acoustic stimulation spectrum affect the spread of frequency-specific adaptation across tonotopically organized areas of auditory cortex. To this end, a model of neural adaptation was used to calculate a response adaptation (RA) index for each individual trial by combining temporal recovery from adaptation with adaptation spread (coadaptation) in auditory cortex (Herrmann et al., 2013a). The RA index reflects normalized expected neural adaptation $(0=$ no adaptation, $1=$ full adaptation) at the time of each tone onset given the history of presented tones (Fig. 1B). The RA index for each trial was based on the individual sequence of tones presented to each participant; that is, independent of the recorded EEG data, and was calculated as follows:

$$
R A_{j+1}=R A_{j}+a \circ\left(1-R A_{j}\right) \times e^{\frac{-\Delta t}{\tau}}
$$


Here, RA corresponds to an $m \times n$ matrix containing expected response adaptation indices for neural populations along the tonotopic gradient of auditory cortex ( $m=$ number of unique tone frequencies in one condition) at each trial's onset ( $n=$ number of trials in one condition). The variable $j$ is the trial index $(j=1 \ldots n), \Delta t$ is the time over which recovery takes place before the subsequent tone onset (here set to the interstimulus interval of 0.3 s; i.e., recovery starts at tone offset), and $\tau$ reflecting the decay in seconds. The operator $\bigcirc$ refers to an entry-wise multiplication (Hadamard product). The column vector $a$ reflects a Gaussian function centered on the presented tone frequency $f_{i}$ of trial $j$ as follows:

$$
a=e^{-0.5 \times\left(\frac{f-f_{i}}{\sigma}\right)^{2}}
$$

where $f$ is a column vector containing the unique tone frequencies $(\log 2$ spaced) of a single condition and $\sigma$ describes the width of the Gaussian function, that is, the spread of adaptation along the tonotopic gradient. The index $i$ refers to the tone frequency presented on trial $j$, that is, it indexes the row entry in $f$ (and RA) on trial $j$. Finally, the RA matrix was reduced to a vector comprising only the expected response adaptation indices for tone frequencies actually presented during the experiment (also excluding trials rejected as artifacts).

To summarize, the relevant parameters of the response adaptation model are $\tau$, reflecting the degree of decay in seconds over which the neural population recovers from adaptation, and $\sigma$, reflecting the degree of adaptation spread in auditory cortex. The other variables were predefined by the experimental setup. RA indices were calculated for each parameter combination ranging from $\tau=0.5-4.5 \mathrm{~s}$ (in steps of $0.1 \mathrm{~s}$ ) and $\sigma=0.1-2.5 \mathrm{SD}$ (in steps of $0.05 \mathrm{SD}$ ).

Data processing: prediction of auditory cortex activation by response adaptation. To investigate the effects of spectral range and spectral spacing on adaptation spread in auditory cortex, a linear function was fitted to single-trial $\mathrm{N} 1$ amplitudes as a function of RA indices.

Linear fits were calculated separately for each $\tau \times \sigma$ combination in each condition (and also separately for the two blocks of presentation of the same condition) and at each electrode and time point. The first five trials in each condition were excluded before fitting. This was done to avoid biasing the fits by the first few trials at the beginning of each presentation block where there is only little adaptation. Linear fitting resulted in a unique slope and intercept value for each $\tau \times \sigma$ parameter combination describing the correlation between RA index and neural activation strength. Here, the slope provides a measure of linear change in neural response magnitude as a function of RA index and the intercept reflects the predicted neural response magnitude assuming no adaptation $($ RA index $=0)$.

For each of the three conditions, slopes and intercepts (for each $\tau \times \sigma$ combination separately) were averaged across the two presentation blocks of the same condition across the samples within the N1 time window (80-120 ms) and across the fronto-central-parietal electrode cluster (Fz, F3, F4, FC3, FC4, Cz, C3, C4, Pz, P3, P4) where neural responses were strongest (Fig. 2; Herrmann et al., 2013a).

The adaptation spread $(\sigma)$ best describing the observed N1 amplitude variations in each condition was independently estimated for each decay parameter $\tau$ as follows. We obtained a measure of observed N1 amplitude at no adaptation (i.e., at full responsiveness) by averaging across singletrial neural responses of the no-adaptation condition (where trials were presented every $10 \mathrm{~s}$ ) and then averaged across the samples within the N1 time window (80-120 ms) and the fronto-central-parietal electrode cluster. The observed N1 magnitude at no adaptation was compared with the intercept value (i.e., predicted $\mathrm{N} 1$ at no adaptation) of each $\sigma$ at a particular $\tau$. Finally, the $\sigma$ best fitting the data was selected where the difference between predicted and observed N1 amplitudes at no adaptation was minimum. In one participant, this minimization resulted in a $\sigma$ at the lower bound of the predefined range $(0.1 \mathrm{SD})$ and the participant was thus excluded from further analysis.

Statistical analysis. Given the constant onset-to-onset interval of $0.5 \mathrm{~s}$, we did not expect changes in recovery time from adaptation $(\tau)$. Therefore, statistical analyses were performed for a fixed $\tau$ of $1.8 \mathrm{~s}$ (Herrmann et al., 2013a), which is well within the range of previous reports regarding temporal decay from adaptation (Mäkelä et al., 1993; Sams et al., 1993; McEvoy et al., 1997).

We first investigated whether the $\mathrm{N} 1$ amplitude systematically varied as a function of RA index. On this account, slopes of the three conditions were separately tested against zero. A one-way repeated measures ANOVA (rmANOVA) was then performed to test for slope differences between conditions (wide-dense, narrow-dense, or wide-sparse). Finally, a one-way rmANOVA was performed to test for differences in $\sigma$ between conditions (wide-dense, narrow-dense, or wide-sparse). Whenever the assumption of sphericity was violated, Greenhouse-Geisser correction was applied (Greenhouse and Geisser, 1959).

\section{Results}

Figure $2 A$ depicts the time courses of the averaged response to each tone in each condition. The strongest modulation of the neural responses was observed in the N1 time window (80-120 $\mathrm{ms})$. Responses were smallest for tone frequencies close to the center (most frequently coadapted) and largest at the edge of a stimulation condition (least frequently coadapted; Fig. 2A, bar graphs; Ulanovsky et al., 2004).

\section{Response adaptation index is predictive of $\mathrm{N} 1$ magnitude}

To determine whether RA indices are predictive of N1 magnitude variations (at $\tau=1.8 \mathrm{~s}$ and individually estimated $\sigma$ ), the slope of each condition was tested against zero. For each condition, the slope was significantly larger than zero (for all three tests; $t_{23}>$ 9.0, $p<0.001$ ). The rmANOVA testing for differences in slopes between conditions was not significant $\left(F_{(2,46)}<2\right)$. These results show that N1 magnitude increased similarly (i.e., N1 amplitude became more negative) in each condition as RA index decreased (Fig. 2B).

In general, linear fits to the $\mathrm{N} 1$ amplitude as function of RA indices were similarly good across conditions. An rmANOVA using the minimized root mean square error of the approximation as dependent measure did not reveal any significant differences between conditions $\left(F_{(2,46)}<1\right)$.

\section{Spectral range but not spectral spacing affects adaptation spread in auditory cortex}

Figure $2 C$ depicts mean $\sigma$ as a function of temporal recovery $(\tau)$ from adaptation that best explains trial-by-trial N1 magnitude variations. Assuming longer recovery time from adaptation (i.e., large $\tau), \sigma$ needs to be tighter to explain the present $\mathrm{N} 1$ data. Similarly, assuming a shorter time period to recover from neural adaptation, $\sigma$ needs to be broader to explain the present $\mathrm{N} 1$ data. These findings highlight the combined contribution of temporal recovery from adaptation and spread of adaptation to frequencyspecific N1 magnitude variations.

Statistical analyses were conducted for a fixed $\tau$ of $1.8 \mathrm{~s}$. The rmANOVA performed to examine differences in neural adaptation spread between conditions revealed a significant main effect $\left(F_{(2,46)}=5.09, p=0.010\right.$; Fig. $\left.2 C\right)$. Adaptation spread was broader (i.e., larger $\sigma$ ) for the wide-dense than for the narrowdense condition (test for spectral range; $t_{23}=2.64, p=0.015$ ) No difference in $\sigma$ was observed between the wide-dense and the wide-sparse condition (test for spectral spacing; $t_{23}=0.32, p=$ 0.754). Broader adaptation spread was also observed for the wide-sparse compared with the narrow-dense condition $\left(t_{23}=\right.$ $2.75, p=0.011$ ).

\section{Discussion}

The present human EEG study investigated whether spread of neural adaptation can become specifically adjusted by tracking statistical properties of the acoustic stimulation spectrum. The 
A

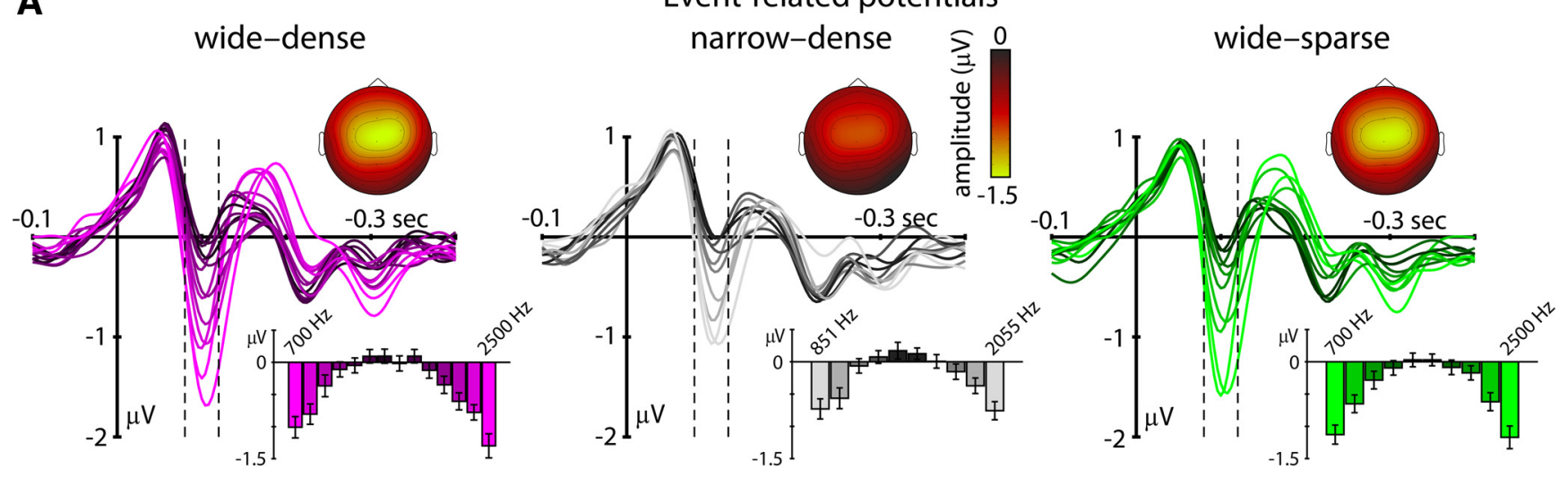

B

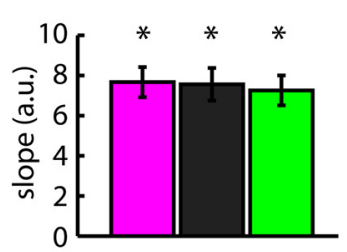

$\tau=1.8 \mathrm{~s} ; \bar{\sigma}=1.11 \mathrm{SD}$

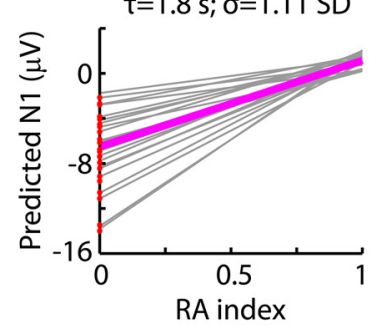

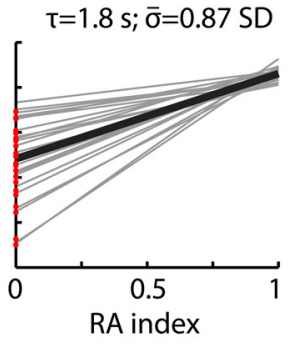

wide-dense

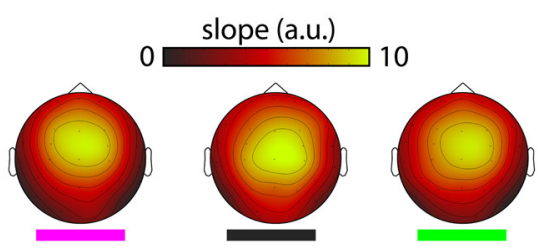

$\tau=1.8 \mathrm{~s} ; \bar{\sigma}=1.13 \mathrm{SD}$

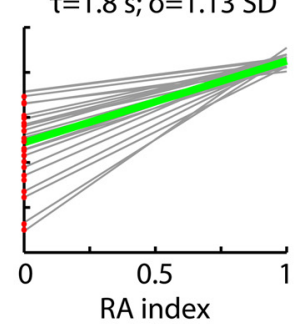

narrow-dense
C Spread of frequency-specific adaptation

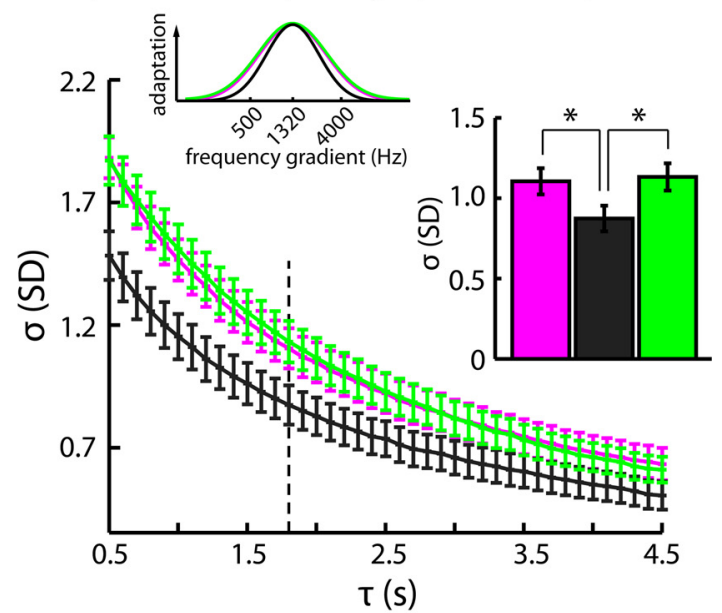

wide-sparse

Figure 2. Event-related potentials. $\boldsymbol{A}$, Time courses of neural responses to each tone frequency in each condition. Bar graphs indicate mean response magnitudes in the N1 time window ( $80-120$ $\mathrm{ms}$; dashed lines). Topographies depict $\mathrm{N} 1$ magnitudes (mean across the two edge frequencies). $\boldsymbol{B}$, Slopes of the linear fits to the N1 magnitude as a function of RA index. The bar graph depicts mean ( $\pm \mathrm{SE}$ ) slopes and the topographies show the respective spatial distribution. ${ }^{*} p<0.001$. Bottom: Predicted N1 magnitudes as a function of RA indices for $\tau=1.8 \mathrm{~s}$ and individually estimated $\sigma$. Mean linear fits are depicted by colored lines and individual fits in gray. Red dots at RA index $=0$ reflect the individual observed N1 magnitudes at no adaptation. $C$, Mean ( \pm SE) $\sigma$ as a function of $\tau$ estimated for the N1 time window (dashed line marks the recovery observed by Sams et al., 1993; $\tau=1.8 \mathrm{~s}$ ). The bar graph and the Gaussian functions show mean adaptation spread ( \pm SE) for each condition at $\tau=1.8 \mathrm{~s} .{ }^{*} p<0.05$.

data show that frequency-specific neural adaptation modulates the auditory cortex N1 component. Critically, N1 neural adaptation is dynamically influenced by the overall spectral range of the acoustic stimulation, but is not affected by the stimulation's spectral spacing.

$\mathrm{N} 1$ magnitude variations in human event-related potentials are known to depend on the frequency and temporal separation between successive stimuli (Butler, 1968; Näätänen et al., 1988; Sams et al., 1993; McEvoy et al., 1997; Herrmann et al., 2013a). Critically, the present study shows that tracking the spectral stimulation history in auditory cortex is dynamic rather than static, such that neural population responses adjust to spectral properties in the ongoing stimulation. Importantly, changes in adaptation spread in tonotopically organized regions of auditory cortex were only observed for variations in spectral range, but not in spectral spacing, of the acoustic stimulation. Therefore, the present results extend previous findings on the dynamics of neural adaptation (Herrmann et al., 2013a, 2013 b) by highlighting the sensitivity of neural populations to changes in the range of the acoustic stimulation spectrum.

Importantly, frequency-specific neural adaptation and adaptation to the distribution properties in the stimulation potentially reflect different mechanisms (Wark et al., 2007). Although frequency-specific adaptation can be accomplished on a singleneuron level, dynamic range adjustments require network coding given the wide range of the stimulation spectrum (Rabinowitz et al., 2011; but see McEvoy et al., 1997, who proposed lateral inhibition as a mechanism of N1 adaptation). Therefore, the N1generating population coding is not only frequency-specific, but flexibly adjusts to the overall spectral range, which in turn affects observable N1 magnitudes.

The present human scalp recordings are consistent with previous results of frequency-specific adaptation in animals (Ulanovsky et al., 2003; Bäuerle et al., 2011; Taaseh et al., 2011), as well as with animal studies reporting response adaptation to statistical properties in the stimulation (Kvale and Schreiner, 2004; Dean et al., 2005, 2008; Wen et al., 2009; Dahmen et al., 2010; Rabinowitz et al., 2011). Neurons along the ascending auditory pathway adjust to statistical properties such as mean and variance and to more complex stimulus distributional properties. Neural dynamic range adaptation in particular has been highlighted in a variety of animal species and sensory modalities, indicating that the input-output relation of neural population coding expands to 
match the range of the stimulation input (Brenner et al., 2000; Rabinowitz et al., 2011; Wen et al., 2012).

Most previous auditory studies investigated dynamic range adjustments to variations in stimulus intensity (Dean et al., 2005, 2008; Wen et al., 2012) or spatial location (Dahmen et al., 2010), whereas the present findings explore neural adjustments to the stimulations' spectral range. Therefore, the present study provides an important extension and links animal studies to stimulus-statistical adaptation in human auditory cortex.

Last, adjustments of neural responses to stimulation statistics requires estimating the parameters of the underlying distribution over time (Wark et al., 2007) and thus critically depends on the temporal evolution and complexity of the stimulation. In the present study, the spectral range could be estimated within a few seconds, which matches previous reports on multiple time scales in neural adaptation (Ulanovsky et al., 2004; Dean et al., 2005; Costa-Faidella et al., 2011). However, adaptation to stimulus statistics has also been observed at much faster time scales (Rabinowitz et al., 2011; Wen et al., 2012) and appears to approach the limits of statistical sampling (Fairhall et al., 2001). When assuming very fast adaptation to statistics in the stimulation spectrum, a trial-by-trial (i.e., almost instantaneous) estimation of distribution parameters and corresponding adjustments would be expected. However, only changes in adaptation spread for manipulations of spectral spacing would have been indicative of such trial-by-trial dynamic adaptation, but these were not observed.

The estimated adaptation spread in the present study was much broader than observed previously, for example, in rat auditory cortex (Taaseh et al., 2011) or in human auditory periphery (as width of critical bands; Herrmann et al., 2013b). Therefore, the present findings may represent a processing stage that already shows substantial across-frequency integration (and therefore adaptation). Therefore, the null finding for spectral spacing could have been due to an insufficient difference in spacing between conditions (dense: 0.141 vs sparse: 0.204 , in $\log 2-$ spacing) to affect the comparably broad spread of adaptation. However, previous estimates of adaptation spread in human auditory cortex yielded similar spread (1.12 SD in Herrmann et al., 2013a) compared with the present study (1.11 and 1.13 SD) despite more sparse spectral spacing $(0.263)$ and identical overall spectral range (Herrmann et al., 2013a). Therefore, based on the strong modulation of adaptation spread by spectral range (suprasecond time scale) rather than spectral spacing (subsecond time scale), we suggest that dynamic range adaptation in human auditory cortex requires integration over a few seconds.

In conclusion, the present findings show that dynamic range adaptation of neural sensitivity in human auditory cortex is directly coupled to the overall spectral range of the acoustic stimulation, which suggests that neural adjustments to spectral stimulus statistics occur over a time scale of multiple seconds.

\section{References}

Bäuerle P, von der Behrens W, Kössl M, Gaese BH (2011) Stimulus-specific adaptation in the gerbil primary auditory thalamus is the result of a fast frequency-specific habituation and is regulated by the corticofugal system. J Neurosci 31:9708-9722. CrossRef Medline

Brenner N, Bialek W, de Ruyter van Steveninck R (2000) Adaptive rescaling maximizes information transmission. Neuron 26:695-702. CrossRef Medline

Butler RA (1968) Effect of changes in stimulus frequency and intensity on habituation of the human vertex potential. J Acoust Soc Am 44:945-950. CrossRef Medline
Costa-Faidella J, Grimm S, Slabu L, Díaz-Santaella F, Escera C (2011) Multiple time scales of adaptation in the auditory system as revealed by human evoked potentials. Psychophysiology 48:774-783. CrossRef Medline

Dahmen JC, Keating P, Nodal FR, Schulz AL, King AJ (2010) Adaptation to stimulus statistics in the perception and neural representation of auditory space. Neuron 66:937-948. CrossRef Medline

Dean I, Harper NS, McAlpine D (2005) Neural population coding of sound level adapts to stimulus statistics. Nat Neurosci 8:1684-1689. CrossRef Medline

Dean I, Robinson BL, Harper NS, McAlpine D (2008) Rapid neural adaptation to sound level statistics. J Neurosci 28:6430-6438. CrossRef Medline

Fairhall AL, Lewen GD, Bialek W, de Ruyter Van Steveninck RR (2001) Efficiency and ambiguity in an adaptive neural code. Nature 412:787-792. CrossRef Medline

Greenhouse SW, Geisser S (1959) On methods in the analysis of profile data. Psychometrika 24:95-112. CrossRef

Herrmann B, Henry MJ, Obleser J (2013a) Frequency-specific adaptation in human auditory cortex depends on the spectral variance in the acoustic stimulation. J Neurophysiol 109:2086-2096. CrossRef Medline

Herrmann B, Henry MJ, Scharinger M, Obleser J (2013b) Auditory filter width affects response magnitude but not frequency specificity in auditory cortex. Hear Res 304:128-136. CrossRef Medline

Kvale MN, Schreiner CE (2004) Short-term adaptation of auditory receptive fields to dynamic stimuli. J Neurophysiol 91:604-612. Medline

Leek MR (2001) Adaptive procedures in psychophysical research. Percept Psychophys 63:1279-1292. CrossRef Medline

Maess B, Jacobsen T, Schröger E, Friederici AD (2007) Localizing preattentive auditory memory-based comparison: Magnetic mismatch negativity to pitch change. Neuroimage 37:561-571. CrossRef Medline

Mäkelä JP, Ahonen A, Hämäläinen MS, Hari R, Ilmoniemi RJ, Kajola M, Knuutila J, Lounasmaa OV, McEvoy L, Salmelin R, Salonen O, Sams M, Simola J, Tesche C, Vasama J-P (1993) Functional differences between auditory cortices of the two hemispheres revealed by whole-head neuromagnetic recordings. Hum Brain Mapp 1:48-56. CrossRef

McEvoy L, Levänen S, Loveless N (1997) Temporal characteristics of auditory sensory memory: neuromagnetic evidence. Psychophysiology 34: 308-316. CrossRef Medline

Näätänen R, Sams M, Alho K, Paavilainen P, Reinikainen K, Sokolov EN (1988) Frequency and location specificity of the human vertex N1 wave. Electroenceph Clin Neurophysiol 69:523-531. CrossRef Medline

Oostenveld R, Fries P, Maris E, Schoffelen JM (2011) FieldTrip: Open source software for advanced analysis of MEG, EEG, and invasive electrophysiological data. Comput Intell Neurosci 156869.

Rabinowitz NC, Willmore BD, Schnupp JW, King AJ (2011) Contrast gain control in auditory cortex. Neuron 70:1178-1191. CrossRef Medline

Ruhnau P, Herrmann B, Schröger E (2012) Finding the right control: The mismatch negativity under investigation. Clin Neurophysiol 123:507512. CrossRef Medline

Sams M, Hari R, Rif J, Knuutila J (1993) The human auditory sensory memory trace persists about 10 sec: neuromagnetic evidence. J Cogn Neurosci 5:363-370. CrossRef Medline

Taaseh N, Yaron A, Nelken I (2011) Stimulus-specific adaptation and deviance detection in the rat auditory cortex. PloS One 6:e23369. CrossRef Medline

Ulanovsky N, Las L, Nelken I (2003) Processing of low-probability sounds by cortical neurons. Nat Neurosci 6:391-398.

Ulanovsky N, Las L, Farkas D, Nelken I (2004) Multiple time scales of adaptation in auditory cortex neurons. J Neurosci 24:10440-10453. CrossRef Medline

Wark B, Lundstrom BN, Fairhall A (2007) Sensory adaptation. Curr Opin Neurobiol 17:423-429. CrossRef Medline

Watkins PV, Barbour DL (2008) Specialized neuronal adaptation for preserving input sensitivity. Nat Neurosci 11:1259-1261. CrossRef Medline

Wen B, Wang GI, Dean I, Delgutte B (2009) Dynamic range adaptation to sound level statistics in the auditory nerve. J Neurosci 29:13797-13808. CrossRef Medline

Wen B, Wang GI, Dean I, Delgutte B (2012) Time course of dynamic range adaptation in the auditory nerve. J Neurophysiol 108:69-82. CrossRef Medline 